Ferrata Storti Foundation

\section{Prognostic impact of prevalent chronic lymphocytic leukemia stereotyped subsets: analysis within prospective clinical trials of the German CLL Study Group}

\author{
Sonia Jaramillo, ${ }^{1}$ Andreas Agathangelidis, ${ }^{2}$ Christof Schneider, ${ }^{1}$ \\ Jasmin Bahlo, ${ }^{3}$ Sandra Robrecht, ${ }^{3}$ Eugen Tausch, ${ }^{1}$ Johannes Bloehdorn, ${ }^{1}$ \\ Manuela Hoechstetter, ${ }^{4}$ Kirsten Fischer, ${ }^{3}$ Barbara Eichhorst, ${ }^{3}$ Valentin Goede, ${ }^{3}$ \\ Michael Hallek, ${ }^{3}$ Hartmut Döhner, ${ }^{1}$ Richard Rosenquist, ${ }^{5}$ Paolo Ghia, ${ }^{6}$ \\ Kostas Stamatopoulos ${ }^{2,5}$ and Stephan Stilgenbauer ${ }^{1}$
}

Volume 105(11):2598-2607

${ }^{1}$ Department of Internal Medicine III, UIm University, UIm, Germany; ${ }^{2}$ Institute of Applied Biosciences, Centre for Research and Technology, Thessaloniki, Greece; ${ }^{3}$ Department I of Internal Medicine and Center of Integrated Oncology Cologne Bonn, University of Cologne, Cologne, Germany; ${ }^{4}$ Department of Hematology, Oncology, Immunology, Palliative Care, Infectious Diseases and Tropical Medicine, München Klinik Schwabing, Munich, Germany; ${ }^{5}$ Department of Molecular Medicine and Surgery, Karolinska Institutet, Stockholm, Sweden and ${ }^{\circledR}$ Università Vita-Salute San Raffaele and IRCCS Ospedale San Raffaele, Milan, Italy

\section{ABSTRACT}

Imost one-third of all patients with chronic lymphocytic leukemia (CLL) express stereotyped B-cell receptor immunoglobulins (BcR IG) 1 and can be assigned to distinct subsets, each with a particular BcR IG. The largest stereotyped subsets are \#1, \#2, \#4 and \#8, associated with specific clinico-biological characteristics and outcomes in retrospective studies. We assessed the associations and prognostic value of these BcR IG in prospective multicenter clinical trials reflective of two different clinical situations: (i) early-stage patients ('watch and wait' arm of the CLL1 trial) $(\mathrm{n}=592)$; (ii) patients in need of treatment, enrolled in three phase III trials (CLL8, CLL10, CLL11), treated with different chemo-immunotherapies $(\mathrm{n}=1,861)$. Subset \#1 was associated with del(11q), higher CLL International Prognostic Index (CLL-IPI) scores and similar clinical course to CLL with unmutated immunoglobulin heavy variable (IGHV) genes (U-CLL) in both early and advanced stage groups. IGHV-mutated (M-CLL) subset \#2 cases had shorter time-to-first-treatment (TTFT) versus other M-CLL cases in the early-stage cohort (hazard ratio [HR]: 4.2, confidence interval [CI]: 2-8.6, $P<0.001$ ), and shorter time-to-next-treatment (TTNT) in the advancedstage cohort (HR: 2, CI: 1.2-3.3, $P=0.005$ ). M-CLL subset \#4 was associated with lower CLL-IPI scores and younger age at diagnosis; in both cohorts, these patients showed a trend towards better outcomes versus other MCLL. U-CLL subset \#8 was associated with trisomy 12. Overall, this study shows that major stereotyped subsets have distinctive characteristics. For the first time in prospective multicenter clinical trials, subset \#2 appeared as an independent prognostic factor for earlier TTFT and TTNT and should be proposed for risk stratification of patients. (Trials registered at clinical trials.gov identifiers: NCT00262782, NCT00281918, NCT2000769522, and NCT01010061).

\section{Introduction}

Chronic lymphocytic leukemia (CLL) is a clinically and biologically heterogeneous disease. ${ }^{1}$ Important prognostic markers include clinical stage (Rai and Binet), presence of deletions in the long arm of chromosome 11 (del(11q)) and in the short arm of chromosome 17(del(17p)), TP53 gene mutations, complex karyotype (CK) as defined by $\geq 3$ chromosomal abnormalities by conventional cytogenetics, markers of tumor load (e.g., thymidine kinase [TK], and 2-microglobulin $[\beta 2 \mathrm{MG}]$, and genetic parameters such as immunoglobulin heavy variable (IGHV) gene somatic 
hypermutation (SHM) status, genomic aberrations, and gene mutations such as SF3B1 and NOTCH1 detected by next-generation sequencing (NGS) $\cdot{ }^{1-4}$ Based on IGHV gene mutational status, CLL can be divided into mutated (MCLL) and unmutated (U-CLL) with indolent and aggressive disease courses, respectively., ${ }^{5,6}$

The combinatorial and junctional diversity of the IGHVIGHD-IGHJ recombination along with the SHM mechanism can lead to the potential synthesis of almost $10^{12}$ different IG. At odds with this diversity, the B-cell receptor (BcR) IG of about one-third of CLL display highly homologous variable heavy complementarity-determining region 3 (VH CDR3), which led to their being grouped into different subsets carrying (almost) identical alias stereotyped BcR IG. ${ }^{7-12}$ Subset \#1 represents approximately $5 \%$ of U-CLL and is characterized by the combination of a heavy chain IGHV1-5-7/IGHD6-19/IGHJ4 gene rearrangement with a light chain IGKV1-39/IGKJ1-2 gene rearrangement. ${ }^{10}$ The IGHV gene bears little or no SHM and the VH CDR3 length is 13 amino acids (aa) long. Subset \#1 is associated with a poor outcome in terms of patient survival and short time-to-first-treatment (TTFT) in comparison to U-CLL using the same IGHV genes. ${ }^{13-15}$ Subset \#2 represents 3\% of all CLL and is defined by the IGHV3-21/IGLV3-21 combination with a short VH CDR3 of 9 aa. ${ }^{10}$ Of note, subset \#2 comprises both U-CLL and MCLL cases and has been associated with an aggressive clinical course, irrespective of SHM status. ${ }^{16-18}$ Subset \#4 is the largest subset of M-CLL, carrying a BcR IG that consists of a heavy chain IGHV4-34/IGHD5-18/IGHJ6 gene rearrangement (20 aa long VH CDR3) and a light chain IGKV2-30/IGKJ1-2 rearrangement. ${ }^{10}$ In previous studies, subset \#4 was associated with indolent disease, enriched in young patients, and a long TTFT..$^{11,15,19}$ Finally, subset \#8 is composed of cases with unmutated IGHV4-39/IGHD613/IGHJ5 gene rearrangements. Furthermore, in a case control study, a strong association with Richter syndrome and poor outcome was reported. ${ }^{8,20}$

Using the data of four prospective multicenter clinical trials of the German CLL study group (GCLLSG), the primary objective of the current study was to search for associations between the most common and best characterized CLL stereotyped subsets (\#1, 2, 4 and 8) and disease characteristics as well as outcome, and also compare these findings against non-subset U-CLL and M-CLL. The secondary objective was to study the prognostic value of subset \#2 irrespective of its IGHV mutational status.

\section{Methods}

\section{Study population}

To assess the prognostic value of the most prevalent CLL subsets, we evaluated patients recruited in four randomized phase III trials conducted by the GCLLSG. None of the patients had received any prior treatment and all had a diagnosis of CLL according to the International Workshop on Chronic Lymphocytic Leukemia criteria. ${ }^{1}$ All trials were approved by the leading ethics committee. Written informed consent was obtained from all patients according to the Declaration of Helsinki. To provide data about the clinical course of newly diagnosed patients not requiring treatment, we evaluated an "early-stage CLL cohort" of 710 asymptomatic Binet stage A patients from the CLL1-trial (clinical trials.gov identifier: NCT00262782). The study compared a 'watch and wait' strategy to upfront fludarabine (F) monotherapy. ${ }^{21}$ In total, 639 (90.0\%) patients were followed with the "watch and wait' approach. Patients treated with F $(71,10.0 \%)$ were excluded from our analyses.

To evaluate the clinical course of patients needing treatment, we evaluated an "advanced-stage cohort" from three phase III trials enrolling patients with CLL requiring front-line treatment. The CLL8-trial (clinical trials.gov identifier: NCT00281918) included 817 fit patients and compared fludarabine and cyclophosphamide (FC) to FC plus rituximab (FCR). ${ }^{22,23}$ The CLL10-study (clinical trials.gov identifiers: NCT00262782 and NCT2000769522) included 561 fit patients and compared FCR to bendamustine and rituximab. ${ }^{24}$ Finally, the CLL11-study (clinical trials.gov identifier: NCT01010061) enrolled 781 unfit patients and compared chlorambucil with or without rituximab or obinutuzumab. ${ }^{25}$

To evaluate the first objective, we classified patients into six groups: subsets \#1, \#2, \#4, \#8, non-subset M-CLL, and U-CLL. To evaluate the second objective, we classified patients into three categories: (i) subset \#2; (ii) IGHV3-21 rearrangement not meeting the subset \#2 criteria ("IGHV3-21"); and (iii) all other cases ("IGHV"). Each group was sub-divided into mutated (" $\mathrm{m}$ ") and unmutated ("u") cases, resulting in six subgroups.

\section{Biological markers and clinical characteristics}

Baseline clinical and laboratory characteristics evaluated for associations and potential prognostic relevance are listed in Tables 1 and 2. Detailed descriptions of the diagnostic methods have been published previously. $3,8,26-30$

\section{Statistical analysis}

Time-to-first-treatment was defined as the time between diagnosis and date of first treatment. For the advanced stage CLI cohort, time-to-next-treatment (TTNT), progression-free survival (PFS) and overall survival (OS) were calculated from randomization to the initiation of subsequent treatment, disease progression or death, and death, respectively. For the early stage cohort, PFS and $O S$ were calculated from diagnosis. Subjects without an event were censored at the time of last assessment. Survival rates were estimated using the Kaplan-Meier method and compared using non-stratified log-rank tests. Hazard ratios and 95\% confidence intervals were calculated using Cox proportional hazards regression model. ${ }^{31}$ All variables that showed significant association with TTFT, TTNT, PFS or OS in univariate Cox regression analyses were included in multivariable analyses applying forward and backward stepwise selection procedures. Adjustments for multiple testing were not applied and all reported $P$-values have an exploratory character. We performed two analyses in both cohorts. In the first one, we compared subsets \#1, 2, 4 and 8 individually to non-subset U-CLL and M-CLL, and in the second one, we evaluated the prognostic value of subset \#2 in terms of mutational status and IGHV3-21 usage.

\section{Results}

\section{Subset assignment and distribution overview}

Of 639 patients from the CLL1 'watch and wait' cohort, immunogenetic data were available for 592 (92.6\%); 402 $(67.9 \%)$ patients carried mutated IGHV genes and 190 (32.1\%) patients expressed unmutated IGHV genes. Eight cases $(1.35 \%)$ were assigned to subset $\# 1$, 16 cases $(2.7 \%)$ to subset \#2, 8 cases $(1.35 \%)$ to subset \#4, and 2 cases $(0.34 \%)$ to subset \#8. With regard to the rest of the cases, $174(29.4 \%)$ belonged to non-subset U-CLL and 384 $(64.9 \%)$ to M-CLL. For the second analysis differentiating the impact of subset \#2, we found 29 cases $(4.9 \%)$ 
Table 1. Baseline characteristics early stage chronic lymphocytic leukemia (CLL) according to subset assignment.

\begin{tabular}{|c|c|c|c|c|c|c|c|}
\hline & Subset \#1 & Subset \#2 & Subset \#4 & Subset \#8 & U-IGHV & m-IEHV & All \\
\hline Target analysis population, $\mathrm{n}(\%)$ & $8(1.35 \%)$ & $16(2.7 \%)$ & $8(1.35 \%)$ & $2(0.337 \%)$ & $174(29.39 \%)$ & $384(64.86 \%)$ & 592 \\
\hline Age at study entry (years) median (range) & $64(56-70)$ & $62(45-75)$ & $58(41-72)$ & $61(56-66)$ & $60(37-75)$ & $60(32-75)$ & $60(32-75)$ \\
\hline Gender, n (\%) male & $6(75.0)$ & $6(37.5)$ & $4(50.0)$ & $1(50.0)$ & $114(65.5)$ & $233(60.7)$ & $364(61.5)$ \\
\hline ECOG performance status median (range) & $0(0-0)$ & $0(0-1)$ & $0(0-0)$ & $0(0-0)$ & $0(0-1)$ & $0(0-2)$ & $0(0-2)$ \\
\hline CLL-IPI risk group, $\mathrm{n}(\%)$ & 8 & 15 & 8 & 2 & 160 & 371 & 564 \\
\hline Low & $0(0.0)$ & $9(60.0)$ & $8(100.0)$ & $0(0.0)$ & $0(0.0)$ & $347(93.5)$ & $364(64.5)$ \\
\hline Intermediate & $6(75.0)$ & $6(40.0)$ & $0(0.0)$ & $2(100.0)$ & $130(81.3)$ & $16(4.3)$ & $160(28.4)$ \\
\hline High & $2(25.0)$ & $0(0.0)$ & $0(0.0)$ & $0(0.0)$ & $26(16.3)$ & $7(1.9)$ & $35(6.2)$ \\
\hline Very high & $0(0.0)$ & $0(0.0)$ & $0(0.0)$ & $0(0.0)$ & $4(2.5)$ & $1(0.3)$ & $5(0.9)$ \\
\hline Leukocyte count (x 109/L) & 36.9 & 23.9 & 21.3 & 10.7 & 23.3 & 20.4 & 21.2 \\
\hline median (range) & $(19.2-128.7)$ & $(4.7-94.4)$ & $(4.7-94.4)$ & $(8.9-12.4)$ & $(4.6-184.0)$ & $(6.3-184.2)$ & $(4.6-184.2)$ \\
\hline Serum thymidine kinase $\mathrm{c}(\mathrm{U} / \mathrm{L})$ & 13.0 & 8.9 & 3.8 & 8.6 & 7.3 & 5.1 & 5.7 \\
\hline median (range) & $(3.5-109.0)$ & $(2.9-23.9)$ & $(2.0-6.5)$ & $(6.5-10.6)$ & $(2.0-56.0)$ & $(1.1-80.0)$ & $(1.1-109.0)$ \\
\hline Serum $\beta 2$-microglobulin(MG/L) & 2.3 & 2.8 & 1.5 & 1.8 & 2.0 & 1.7 & 1.8 \\
\hline median (range) & $(1.6-6.7)$ & $(1.1-4.4)$ & $(1.0-3.4)$ & $(1.3-2.3)$ & $(0.2-9.4)$ & $(0.4-8.9)$ & $(0.2-9.4)$ \\
\hline Deletion 11Q by FISH, n (\%) & $3(37.5)$ & $1(6.7)$ & $0(0.0)$ & $0(0.0)$ & $32(19.6)$ & $5(1.3)$ & $39(6.9)$ \\
\hline Deletion 17P by FISH, n (\%) & $0(0.0)$ & $0(0.0)$ & $0(0.0)$ & $0(0.0)$ & $7(4.3)$ & $8(2.1)$ & $15(2.7)$ \\
\hline Trisomy 12 by FISH, n (\%) & $1(12.5)$ & $0(0.0)$ & $1(12.5)$ & $2(100.0)$ & $33(20.0)$ & $20(5.3)$ & $51(9.1)$ \\
\hline Deletion $13 \mathrm{Q}$ by FISH, n (\%) & $3(37.5)$ & $9(60.0)$ & $2(25.0)$ & $1(50.0)$ & $87(52.4)$ & $250(67.2)$ & $295(52.4)$ \\
\hline NOTCH1 MUTATED, n (\%) & $2(25.0)$ & $1(6.7)$ & $0(0.0)$ & $1(50.0)$ & $10(6.6)$ & $4(1.1)$ & $18(3.4)$ \\
\hline SF3B1 MUTATED, n (\%) & $2(25.0)$ & $8(53.3)$ & $0(0.0)$ & $0(0.0)$ & 17 (11.3) & $11(3.1)$ & $38(7.1)$ \\
\hline Richter syndrome status, n (\%) & 0 & 0 & 0 & 0 & $4(2.3)$ & $5(1.3)$ & $9(1.5)$ \\
\hline
\end{tabular}

n: number of cases; u: unmutated; m: mutated; IGHV: non-subset immunoglobulin heavy variable; ECOG: Eastern Cooperative Oncology Group; IPI: International Prognostic Index;FISH: fluorescence in situ hybridization.

expressing the IGHV3-21 gene. Among those, 16 cases $(2.7 \%)$ belonged to subset \#2, of which 6 cases $(1 \%)$ were u-subset \#2 and 10 cases $(1.7 \%)$ were m-subset \#2. The remaining 13 cases $(2.2 \%)$ carried heterogeneous (nonsubset) BcR IG (IGHV3-21). Of these, 3 (0.5\%) were uIGHV3-21 and $10(1.7 \%)$ were m-IGHV3-21 (Online Supplementary Table S1).

For patients requiring front-line treatment from the CLL8, CLL10 and CLL11 trials or advanced stage cohort, immunogenetic data were available in 1,861 cases $(86.2 \%)$, of which $39(2.1 \%)$ were assigned to subset \#1, $61(3.3 \%)$ to subset \#2, $11(0.6 \%)$ to subset \#4, and 16 $(0.9 \%)$ to subset \#8. The remaining 1,734 cases did not belong to these subsets; 1,088 (58.4\%) were U-CLL and $646(34.7 \%)$ cases were M-CLL. For the second analysis, we identified 126 cases (6.8\%) expressing IGHV3-21 BcR IG. Of these, 61 (3.3\%) were assigned to subset \#2, 18 $(1.0 \%)$ were u-subset \#2, and $43(2.3 \%)$ m-subset \#2, while $65(3.5 \%)$ cases belonged to the group expressing heterogeneous IGHV3-21. Of these, 35 (1.9\%) were u-IGHV3-21 and 30 (1.6\%) were m-IGHV3-21 (Online Supplementary Table S2).

\section{Association of subsets with clinical and biological characteristics}

Clinical and biological characteristics in the early stage CLL cohort according to subset assignment and IGHV mutational status are listed in Table 1. Despite the size of the overall cohort, small case numbers in patient subgroups precluded valid statistical assessment, but a pattern similar to that observed in the advanced stage cohort (see below) was seen. Subset \#1 showed a high frequency of $\operatorname{del}(11 \mathrm{q})$ and NOTCH1 mutations, subset \#2 had a lower rate of male patients and a high frequency of SF3B1 mutations, and subset \#4 were younger at study entry. Both cases of subset \#8 had trisomy 12 and intermediate risk according to CLL-IPI, and neither of them displayed a Richter transformation. In the second analysis, when we compared m-subset \#2 with m-IGHV3-21 and m-IGHV, we found an imbalance of SF3B1 mutations $(P<0.001)$ and a higher presence of $\operatorname{del}(11 q)(P=0.014)$.

The clinical and biological characteristics of the advanced stage cohort according to BcR IG stereotype and IGHV SHM status are listed in Table 2. Subset \#1 together with subset \#8 showed higher frequencies of NOTCH1 mutations and del(11q). Subset \#2 demonstrated an enrichment for SF3B1 mutations. As in the early stage cohort, subset \#4 were younger at diagnosis. Finally, subset \#8 showed a higher frequency of Richter transformation. In the second analysis, we observed a higher prevalence of $\operatorname{del}(13 q)$ in u-subset \#2 (88.9\%) compared to the u-IGHV3-21 (46.9\%) and u-IGHV patients $(45.1 \%$, $P=0.001)$. A higher frequency of SF3B1 mutations was also seen in subset \#2 patients independently of the IGHV SHM status (u-subset \#2, $41.7 \%$ and m-subset \#2, 46.7\%, respectively). $\mathrm{M}$-subset \#2 showed a higher prevalence of del(11q) compared to m-IGHV3-21 and m-IGHV (32.5\% vs. $13.3 \%$ vs. $5.5 \%$, respectively).

\section{Association between stereotyped subsets and clinical outcomes}

After a median observation time of 97.8 (range, 2.5166.9) months (ms) of the early stage cohort, there were 246, 362 and 106 events for TTFT, PFS, and OS, respec- 
Table 2. Baseline characteristics of the advanced stage chronic lymphocytic leukemia (CLL) cases according to subset classification.

\begin{tabular}{|c|c|c|c|c|c|c|c|}
\hline & Subset \#1 & Sulbset \#2 & Subset \#4 & Subset \#8 & U-IGHV & m-IERV & All \\
\hline Target analysis population, $\mathrm{n}(\%)$ & $39(2.09 \%)$ & $61(3.28 \%)$ & $11(0.59 \%)$ & $16(0.86 \%)$ & $1088(58.46 \%)$ & $646(34.71 \%)$ & 1,861 \\
\hline Age at study entry (years) median (range) & $66(43-84)$ & $66(48-88)$ & $52(42-74)$ & $67(35-84)$ & $65(30-90)$ & $65(36-89)$ & $65(30-90)$ \\
\hline Gender, n (\%) Male & $24(61.5)$ & $36(59.0)$ & $6(54.5)$ & $8(50.0)$ & $775(71.2)$ & $437(67.6)$ & $1286(69.1)$ \\
\hline ECOG performance status median (range) & $0.5(0-2)$ & $1(0-2)$ & $1(0-1)$ & $0.5(0-2)$ & $1(0-3)$ & $0(0-3)$ & $1(0-3)$ \\
\hline Binet stage, n (\%) & 39 & 61 & 11 & 16 & 1087 & 646 & 1860 \\
\hline A & $6(15.4)$ & $7(11.5)$ & $1(9.1)$ & $2(12.5)$ & $180(16.6)$ & $112(17.3)$ & $308(16.6)$ \\
\hline B & $20(51.3)$ & $33(54.1)$ & $6(54.5)$ & $11(68.8)$ & $568(52.3)$ & $243(37.6)$ & $881(47.4)$ \\
\hline $\mathrm{C}$ & $13(33.3)$ & $21(34.4)$ & $4(36.4)$ & $3(18.8)$ & $339(31.2)$ & $291(45.0)$ & $671(36.1)$ \\
\hline CLL-IPI risk group, n (\%) & 38 & 57 & 11 & 16 & 1029 & 593 & 1744 \\
\hline Low & $1(2.6)$ & $18(31.6)$ & $7(63.6)$ & $0(0.0)$ & $0(0.0)$ & $252(42.5)$ & $278(15.9)$ \\
\hline Intermediate & $10(26.3)$ & $19(33.3)$ & $3(27.3)$ & $4(25.0)$ & $388(37.7)$ & $228(38.4)$ & $652(37.4)$ \\
\hline High & $26(68.4)$ & $18(31.6)$ & $1(9.1)$ & $12(75.0)$ & $534(51.9)$ & $105(17.7)$ & $696(39.9)$ \\
\hline Very high & $1(2.6)$ & $2(3.5)$ & $0(0.0)$ & $0(0.0)$ & $107(10.4)$ & $8(1.3)$ & $118(6.8)$ \\
\hline Leukocyte count $($ x 109/L) & 104.7 & 72.3 & 73.5 & 56.4 & 85.3 & 65.9 & 79.1 \\
\hline median (range) & $(13.7-375.0)$ & $(3.1-492.0)$ & $(19.2-144.1)$ & $(12.9-118.0)$ & $(0.2-867.0)$ & $(3.1-741.9)$ & $(0.2-867.0)$ \\
\hline Serum thymidine kinase $c(\mathrm{U} / \mathrm{L})$ & 26.6 & 20.2 & 10.2 & 55.9 & 19.2 & 11.3 & 16.5 \\
\hline median (range) & $(0.5-330.6)$ & $(0.0-163.0)$ & $(4.1-36.3)$ & $(0.5-165.3)$ & $(0.0-848.0)$ & $(0.0-970.0)$ & $(0.0-970)$ \\
\hline Serum $\beta 2$-microglobulin (MG/L) & 3.4 & 3.0 & 2.2 & 3.8 & 3.0 & 2.8 & 3.0 \\
\hline median (range) & $(0.6-8.1)$ & $(0.0-9.8)$ & $(0.5-5.1)$ & $(0.3-8.1)$ & $(0.0-17.8)$ & $(0.0-12.2)$ & $(0.0-17.8)$ \\
\hline Deletion 17P by FISH, n (\%) & $1(2.6)$ & $0(0.0)$ & $0(0.0)$ & $0(0.0)$ & $79(7.5)$ & $21(3.4)$ & $101(5.6)$ \\
\hline Deletion 11Q by FISH, n (\%) & $17(43.6)$ & $18(31.0)$ & $0(0.0)$ & $6(37.5)$ & $316(29.8)$ & $37(6.0)$ & $378(21.0)$ \\
\hline Trisomy 12 by FISH, n (\%) & $4(10.3)$ & $2(3.4)$ & $1(9.1)$ & $11(68.8)$ & $163(15.4)$ & $80(13.0)$ & $222(12.3)$ \\
\hline Deletion $13 \mathrm{Q}$ by FISH, n (\%) & $23(59.0)$ & $49(84.5)$ & $6(54.5)$ & $2(12.5)$ & $477(45.1)$ & $409(66.3)$ & $648(36.0)$ \\
\hline TP53 mutated, n (\%) & $1(3.8)$ & $2(4.8)$ & $0(0.0)$ & $0(0.0)$ & $103(13.6)$ & $30(6.9)$ & $136(10.6)$ \\
\hline NOTCH1 mutated, n (\%) & $4(16.0)$ & $2(4.8)$ & $0(0.0)$ & $5(38.5)$ & $154(20.5)$ & $20(4.7)$ & $185(14.6)$ \\
\hline SF3B1 mutated, $\mathrm{n}(\%)$ & $0(0.0)$ & $19(45.2)$ & $0(0.0)$ & $0(0.0)$ & $131(17.4)$ & $52(12.1)$ & $202(15.9)$ \\
\hline Richter syndrome status, n (\%) & $1(2.6)$ & $2(3.3)$ & 0 & $1(6.3)$ & $35(3.2)$ & $14(2.2)$ & $53(2.8)$ \\
\hline
\end{tabular}

n: number of cases; u: unmutated; m: mutated; IGHV: non-subset immunoglobulin heavy variable; ECOG: Eastern Cooperative Oncology Group; IPI: International Prognostic Index;FISH: fluorescence in situ hybridization.

tively. TTFT was significantly longer in M-CLL as compared to U-CLL $(P<0.001)$. Subset \#4 had a longer TTFT compared to subsets \#1, \#2 and \#8 $(P=0.002)$, but similar to M-CLL $(P=0.338)$ (Figure $1 \mathrm{~A})$. Therapy was needed only for 1 of 8 patients assigned to subset \#4. Statistically, there was no difference in TTFT between subsets \#1, 2 and 8 , and U-CLL ( $P=0.204$ ) (Figure 1A). Regarding PFS, a significant difference was observed between M-CLL and U-CLL $(P<0.001)$. Subset \#4 had a longer PFS than subsets $\# 1,2$ and $8(P=0.014)$ that was similar to M-CLL cases $(P=0.249)$, with only two patients progressing. Subsets \#1, 2, 8 and U-CLL had a similar PFS $(P=0.198)$ (Figure 1B). Regarding OS, M-CLL had a significantly longer time to event compared to U-CLL $(P<0.001)$. Subsets \#1, 2, 4, and 8 showed no statistically significant differences in terms of OS; however, the OS curves followed a similar pattern to that observed for PFS and TTFT with subsets \#1, 2, and 8, showing more events as compared to subset \#4 where no incidents of death were observed (Figure 1C).

During a median observation time of $55.1 \mathrm{~ms}$ of the advanced stage cohort, there were 1,847, 1,258, 739 and 512 events for TTFT, PFS, TTNT, and OS, respectively. TTFT was longer for M-CLL as compared to U-CLL $(P<0.001)$. Subset \#4 had a similar TTFT to that of M-CLL that was longer as compared to subsets \#1, 2 and 8
$(P=0.009)$. Subsets \#1, 2 and 8 had a similar TTFT as UCLL (Figure 2A). Regarding PFS, M-CLL had a longer time to event compared to U-CLL $(P<0.001)$. Subset \#4 patients had a longer PFS compared to subsets \#1, 2, and 8; however; this difference was not statistically significant. Subset \#8 had a shorter PFS compared to subsets \#1, 2 and U-CLL $(P=0.042)$ (Figure 2C). Regarding TTNT, M-CLL had a longer time to event compared to U-CLL $(P<0.001)$. No subset \#4 patient needed subsequent therapy during the observation time and TTNT was longer as compared to subsets \#1, 2, and $8(P=0.027)$. Subset \#4 also had a longer TTNT compared to M-CLL without reaching statistical significance $(P=0.058)$ (Figure $2 \mathrm{~B})$. Regarding $O S$, we observed a significant difference between M-CLL and U-CLL $(P<0.001)$. None of the subset \#4 patients died before time of analysis; subset \#4 patients had a longer $O S$ than subsets \#1, 2 and $8(P=0.011)$ (Figure 2D).

\section{Cases expressing IGHV3-21: subset \#2 and others}

In the cohort of early stage CLL patients, m-subset \#2 had a significantly shorter TTFT compared to m-IGHV321 and $\mathrm{m}$-IGHV patients $(P<0.001)$. While m-subset \#2 had an outcome similar to that of the u-IGHV group $(P=0.794)$, m-IGHV3-21 was comparable to m-IGHV (Figure 3A). Similarly, PFS was shorter in m-subset \#2 
A

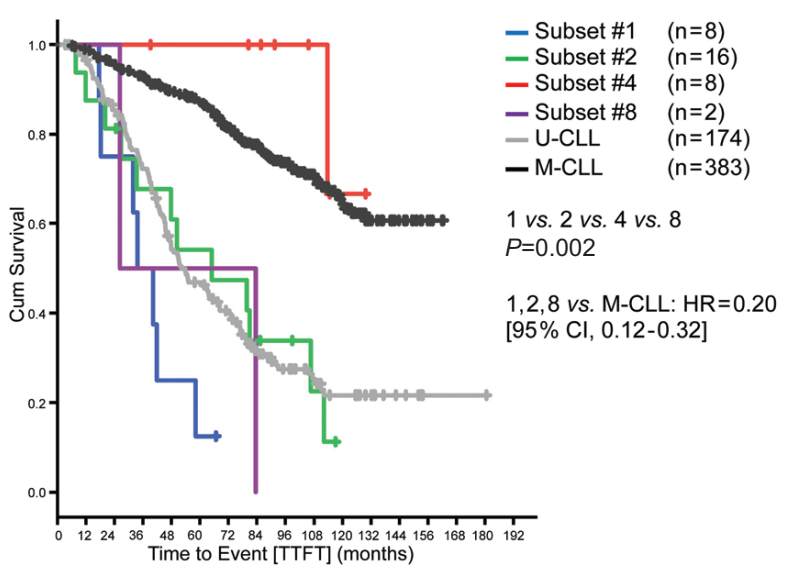

C

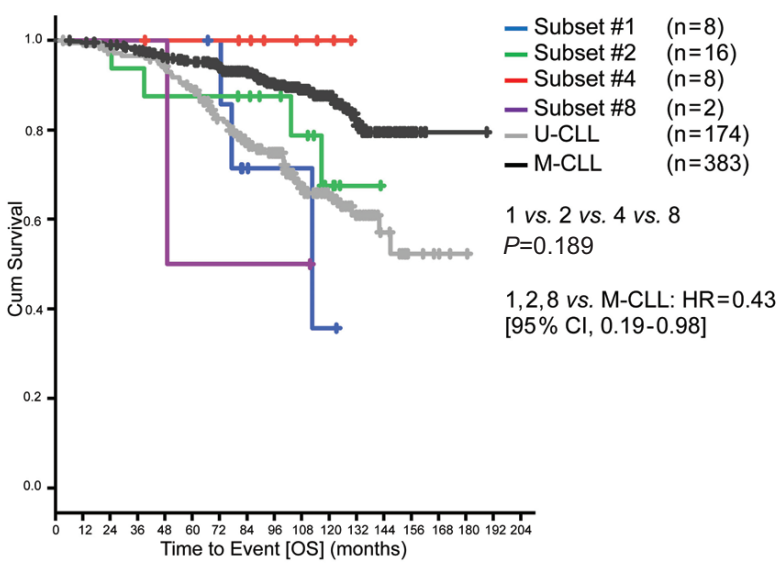

compared to m-IGHV3-21 and m-IGHV cases, but did not reach statistical significance $(P=0.161)$. However, the u-subset \#2 cases had a shorter time to event compared to u-IGHV3-21 and u-IGHV $(P=0.006)$ (Figure 3B). Regarding $\mathrm{OS}$, there was no significant difference between $\mathrm{m}$-subset $\# 2$, m-IGHV3-21 and m-IGHV $(P=0.221)$ (Figure 3C); however, small event numbers precluded robust comparisons despite the long observation time.

In the advanced stage cohort, $\mathrm{m}$-subset $\# 2$ cases had a shorter TTFT compared to m-IGHV3-21 and m-IGHV $(P=0.056)$, and showed similar behavior to that of the uIGHV group $(P=0.215)$. u-IGHV3-21 patients showed a shorter TTFT compared to u-subset \#2 and u-IGHV cases $(P=0.050)$ (Figure 4A). Similarly, m-subset \#2 cases showed a shorter TTNT than m-IGHV3-21 and m-IGHV patients $(P=0.001)$. Furthermore, $\mathrm{m}$-subset $\# 2$ had a similar clinical course to u-IGHV cases in terms of TTFT $(P=0.401) \quad$ (Figure 4B). No difference was observed between u-IGHV3-21, u-subset $\# 2$ and u-IGHV ( $P=0.536)$. In the PFS analysis, $\mathrm{m}$-subset \#2 showed a significantly shorter PFS compared to m-IGHV3-21 and m-IGHV $(P=0.020)$ (Figure 4C). No difference was observed between $\mathrm{m}$-subset $\# 2$ and $\mathrm{u}$-IGHV $(P=0.065)$ (Figure $4 \mathrm{C}$ ). Regarding OS, we observed no significant difference in terms of survival rate at 5 years between $\mathrm{m}$-subset $\# 2$, $\mathrm{m}$ IGHV3-21 and m-IGHV or u-subset \#2, u-IGHV3-21 and u-IGHV ( $P=0.717, P=0.752$, respectively) (Figure 4D).

\section{Multivariate analyses}

Due to the resemblance in all clinical outcomes, the sim-
B

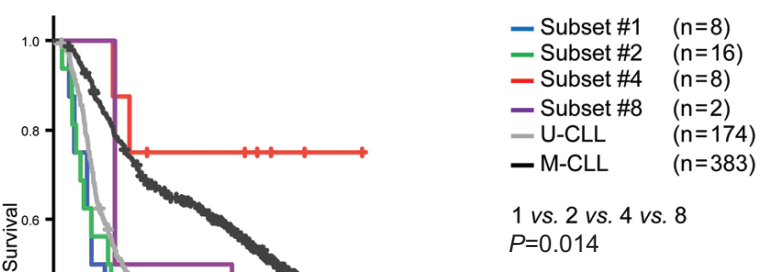

$1,2,8$ vs. M-CLL: $H R=0.44$ $[95 \% \mathrm{Cl}, 0.27-0.71]$
Figure 1. Outcomes of early stage chronic lymphocytic leukemia (CLL) cases according to subset classification. (A) Median time-to-first-treatment (TTFT) subset \#1: 33.7 months, subset \#2: 65.0 months, subset \#4: not reached, subset \#8: 26.3 months, U-CLL: 52.2 months, M-CLL: not reached. (B) Median progression-free survival (PFS) from diagnosis subset \#1: 15.9 months, subset \#2: 22.8 months, subset \#4: not reached, subset \#8: 25.7 months, U-CLL: 28.8 months, M-CLL: 92.6 months. (C) Median overall survival (OS) from diagnosis subset \#1: 112.5 months, subset \#2: not reached, subset \#4: not reached, subset \#8: 49.0 months, U-CLL: not reached, M-CLL: not reached; Cum: cumulative. ilarities between subsets (Figures 1 and 2) and the small size of the subset population, we grouped subsets \#1, 2 and 8 together to be able to analyze their relevance as a group in the clinical outcomes of interest. Subset \#4 was analyzed as part of M-CLL for the same reasons. We compared this group of subsets to M-CLL and U-CLL. In a supplementary analysis, we also compared subset \#2 to subsets \#1 and 8 as well as to M-CLL and U-CLL.

A Cox multivariate regression analysis was chosen to adjust for the variables that were significant in the univariate analysis.

For the early stage cohort, the variables included in the final model for TTFT are listed in Table 3. M-CLL and subset \#4 had a longer TTFT compared to subsets \#1,2 and 8 $(P<0.01)$. In the supplementary analysis, subset $\# 2$ alone showed the same differences (Online Supplementary Table S3). M-CLL and subset \#4 had also a longer PFS and OS compared to the group of subsets (hazard ratio [HR]:0.44, confidence interval [CI]: $0.272-0.712, P=0.001$ and HR:0.431, CI: 0.19-0.978, $P<0.044$, respectively). In a second multivariate analysis, we tested for the independent value of the $\mathrm{m}$-subset \#2 subgroup, including the variables listed in Table 4. This identified $\mathrm{m}$-subset \#2 as an independent adverse prognostic marker for shorter TTFT as compared to m-IGHV $(P<0.001)$ (Table 4). For the PFS multivariate analysis, we observed no differences between m-subset \#2 and m-IGHV or m-IGHV3-21 and m-IGHV ( $P=0.556)$.

In the advanced stage cohort, the TTNT final model found a significant difference between M-CLL and subsets 
A

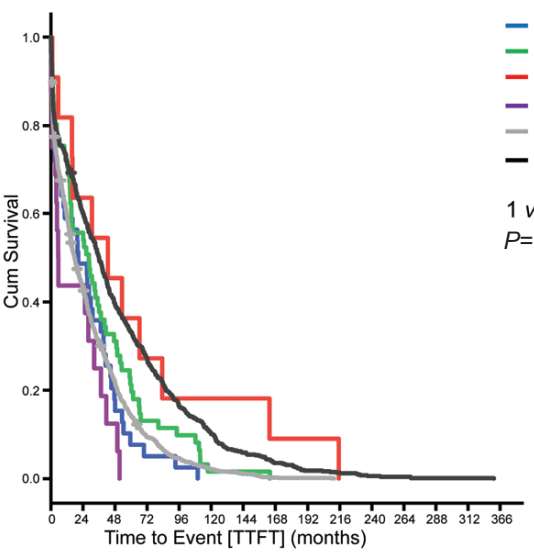

C
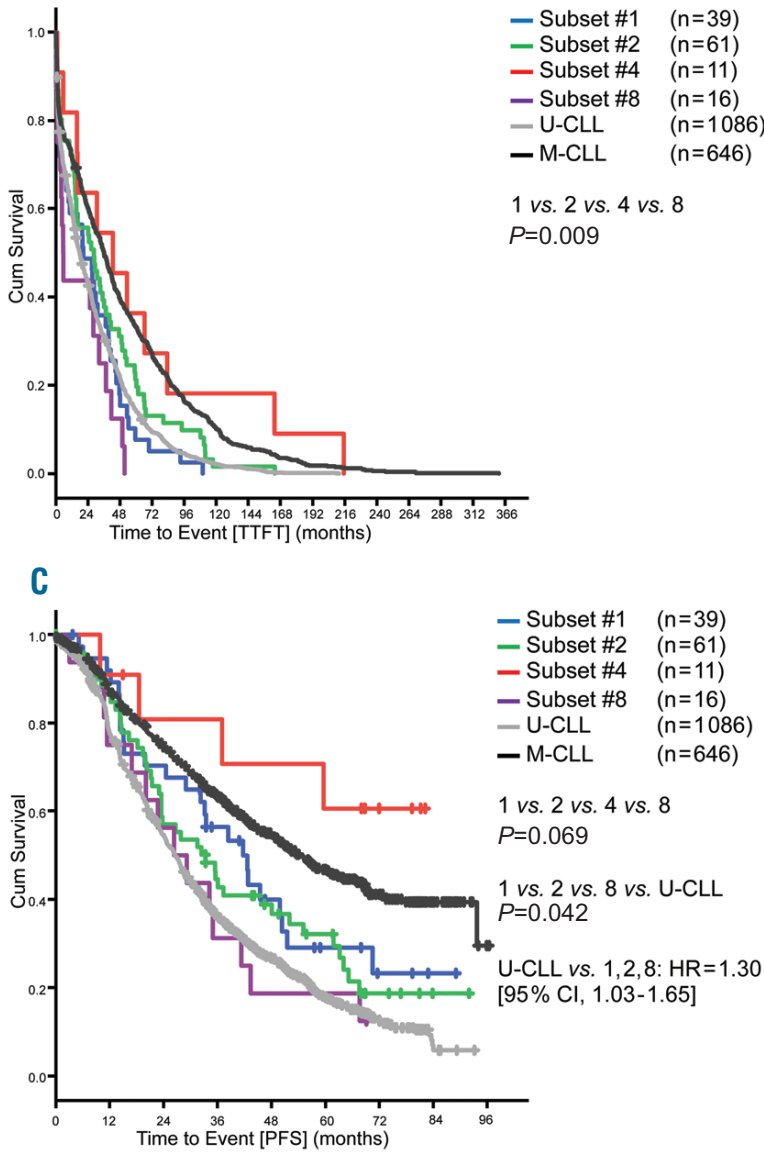

B

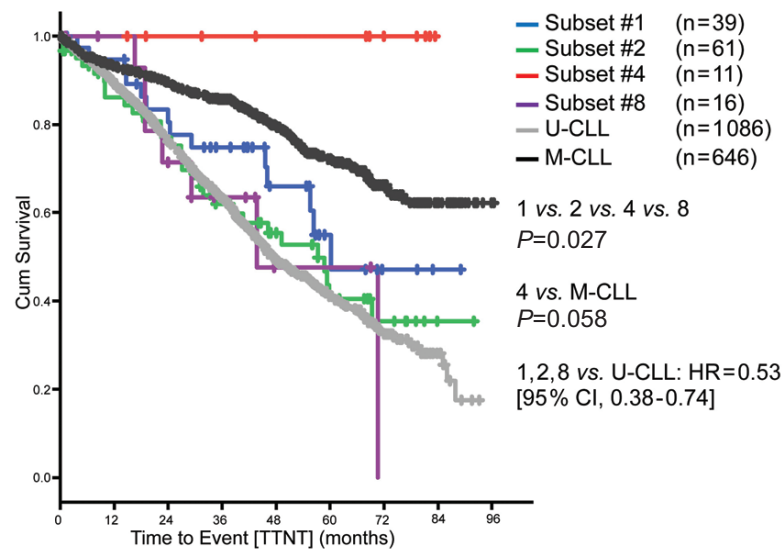

D

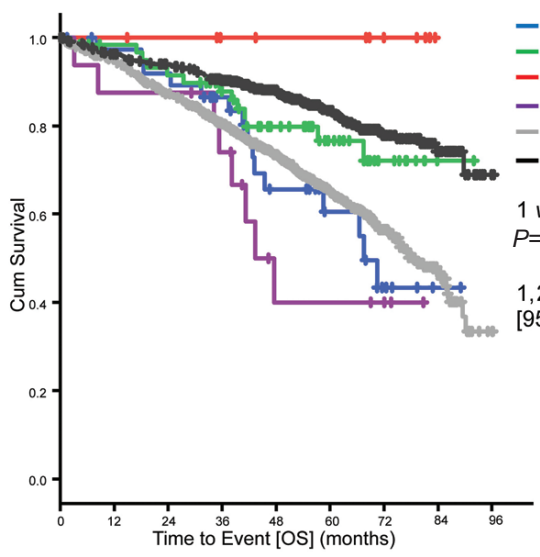

Figure 2. Outcomes of advanced stage chronic lymphocytic leukemia (CLL) cases according to subset classification. (A) Median time-to-first-treatment (TTFT) for the subset \#1: 20.6 months, subset \#2: 28.5 months, subset \#4: 42.8 months, subset \#8: 5.5 months, U-CLL: 17.8 months, M-CLL: 36.1 months. (B) Median timeto-next-treatment (TTNT) for the subset \#1: 60.2 months, subset \#2: 57.3 months, subset \#4: not reached, subset \#8: 43.7 months, U-CLL: 47.4 months, M-CLL: not reached. (C) Median progression-free survival (PFS) for subset \#1: 41.7 months, subset \#2: 33.3 months, subset \#4: not reached, subset \#8: 26.3 months, U-CLL: 26.5 months, M-CLL: 54.7 months. (D) Median overall survival (OS) for subset \#1: 67.5 months, subset \#2: not reached, subset \#4: not reached, subset \#8: 43.3 months, U-CLL: 78.9 months, M-CLL: not reached; Cum: cumulative.

\#1, 2 and 8 (HR: 0.53, CI: 0.38-0.74, $P<0.001)$. This same pattern was also seen when M-CLL was compared to subset \#2 alone; however, this difference was not statistically significant $(P=0.052)$ (Online Supplementary Table S4). UCLL had a significantly shorter PFS compared to subsets \#1, 2 and 8 ( $P=0.028)$ as opposed to M-CLL that showed a significantly longer PFS $(P=0.002)$ (Online Supplementary Table S5). In the supplementary analysis, U-CLL also had a shorter PFS compared to subset \#2 $(P<0.05)$ (Online Supplementary Table S6). In the OS final model, we observed a significant difference between M-CLL and subsets $\# 1,2$ and $8(P=0.002)$. In the second analysis, we found a statistically significant difference between TTNT in m-subset \#2 and m-IGHV $(P=0.005)$ (Online Supplementary Table S7). With regards to PFS, no differences were found between m-subset \#2 and m-IGHV $(P=0.122)$.

\section{Discussion}

Our study evaluated the impact of BcR IG stereotype on four relevant clinical outcomes (TTFT, TTNT, PFS, and
OS) with a sufficient follow-up period in four major CLL stereotyped subsets, i.e., subsets \#1, 2, 4 and 8. Two different clinical scenarios were considered in the present analysis: early stage patients with CLL included in the 'watch and wait' arm of the CLL1 study, and advanced stage, treatment-naive patients with CLL treated within three clinical trials of the GCLLSG. The analysis was performed in patients within prospective clinical trials, where the information regarding the most relevant clinical and genetic characteristics of subsets \#1,2, 4 and 8 could be analyzed in a controlled setting with mature follow-up.

Subset \#1 had a similar prognosis to U-CLL in both early stage and advanced stage CLL patients. These findings were consistent with previous publications, where this subset has been associated with a poor prognosis in terms of survival and TTFT even when compared to CLL with the same IGHV genes but a heterogenous BcR IG. ${ }^{14}$

Subset \#2 patients are known to have a shorter TTFT as well as OS regardless of IGHV mutational status. ${ }^{15,33,34}$ In our study, we observed this same behavior in both early and advanced stage CLL. Furthermore, consistent with previous publications, we found that subset \#2 cases had a higher rate of $S F 3 B 1$ mutations in both the IGHV mutat- 
ed and unmutated state, which, however, does not seem to explain the unfavorable clinical prognosis. ${ }^{29,32,33}$

It has been reported that subset \#2 has a higher progression rate to disease requiring treatment and also a shorter OS. ${ }^{15,33,34}$ In our analysis, in both early and advanced stage CLL, m-subset \#2 showed higher presence of del(11q) and unfavorable prognosis compared to other IGHV-mutated CLL cases. This difference was also observed in a multivariate analysis, where $\mathrm{m}$-subset \#2 patients had a worse outcome compared to m-IGHV with regards to TTFT in early stage CLL, and TTNT in advanced stage CLL patients. Our data showed for the first time in clinical studies that subset \#2 is an independent prognostic factor for earlier TTFT in early stage CLL. There was a significant difference in TTFT between mutated subsets \#2 and $\mathrm{m}-\mathrm{IGHV}$ in both the univariate and multivariate analysis. This finding was also based on multiple validation analyses that corroborated our results. Therefore, it is safe to say that the conclusion is valid despite the small sample. No significant differences were observed between m-subset \#2, m-IGHV3-21 and m-IGHV in early or advanced stage CLL in terms of PFS or OS. This implies that patients
A

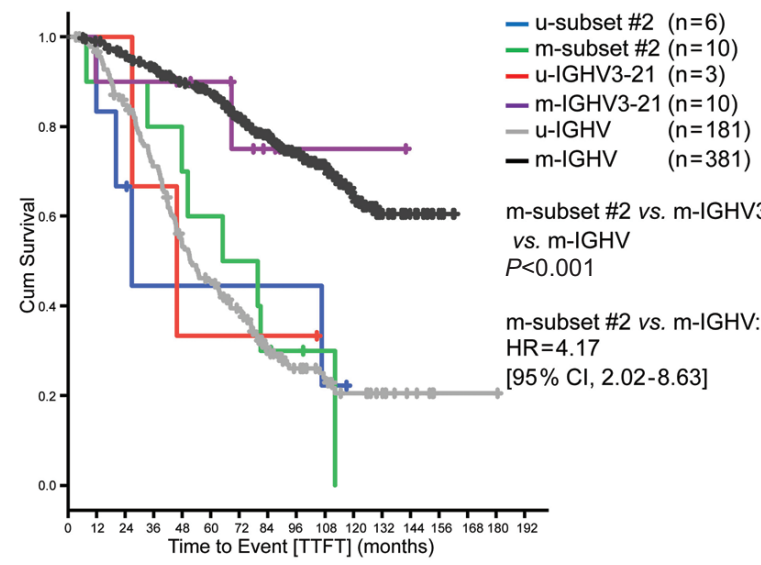

C

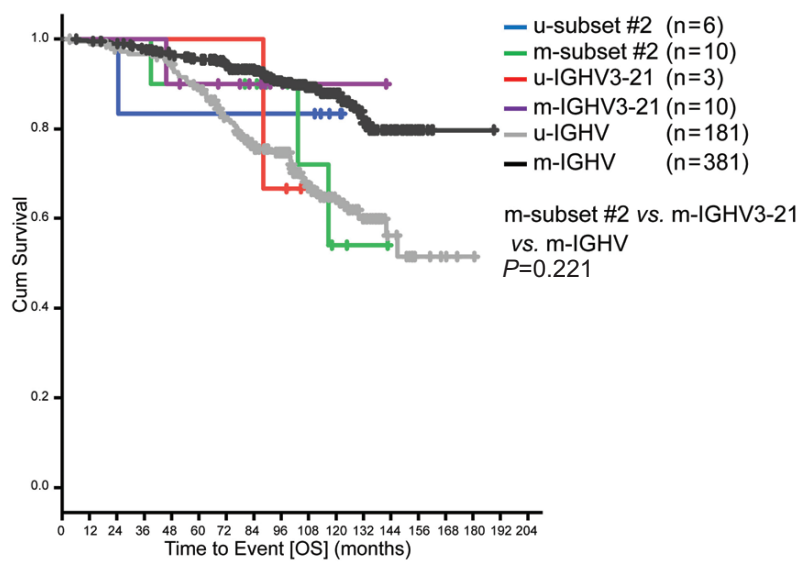

B

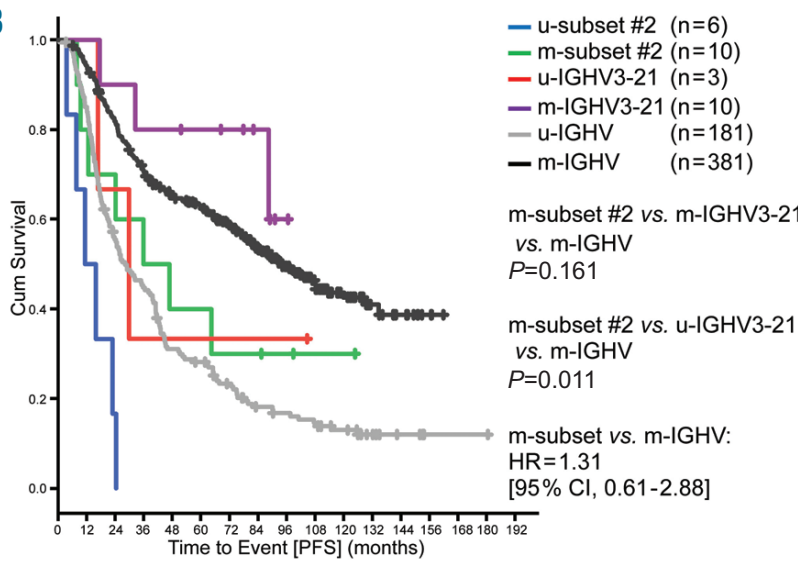

Figure 3. Outcomes of early stage chronic lymphocytic leukemia (CLL) cases according to subset \#2 classification and IGHV mutation. (A) Median time-to-first-treatment (TTFT) for u-subset \#2: 26.8 months, msubset \#2: 65.0 months, u-HV3-21: 45.7 months, m-HV3-21: not reached, u-IGHV: 51.4 months, m-IGHV: not reached. (B) Median progression-free survival (PFS) from diagnosis for u-subset \#2: 11.2 months, m-subset \#2: 35.7 months, u-HV3-21: 29.8 months, m-HV321: not reached, u-IGHV: 28.1 months, m-IGHV: 92.6 months. (C) Median overall survival (OS) from diagnosis for u-subset \#2: not reached, m-subset \#2: not reached, u-HV3-21: not reached, m-HV3-21: not reached, u-IGHV: not reached, m-IGHV: not reached; Cum: cumulative.

Table 3. Time-to-first-treatment (TTFT) Cox regression in early stage chronic lymphocytic leukemia (CLL) subsets \# 1, 2 and 8.

\begin{tabular}{|c|c|c|c|c|c|}
\hline \multirow{2}{*}{$\begin{array}{l}\text { Cox regression } \\
\text { TIF }\end{array}$} & \multirow{2}{*}{$\begin{array}{l}\text { Univariable } \\
\text { comparison }\end{array}$} & \multirow[t]{2}{*}{ Hazard ratio } & \multicolumn{2}{|c|}{$95 \%$ confidence interval } & \multirow[t]{2}{*}{$\boldsymbol{P}$} \\
\hline & & & Lower & Upper & \\
\hline \multicolumn{6}{|c|}{ Subset and IGHV analysis group } \\
\hline U-CLL & vs. Subset \#1,2,8 & 0.812 & 0.504 & 1.308 & 0.392 \\
\hline M-CLL and subset \#4 & vs. Subset \#1,2,8 & 0.195 & 0.119 & 0.318 & $<0.001$ \\
\hline \multicolumn{6}{|c|}{ Deletion in $17 p$} \\
\hline Yes & vs. no & 2.332 & 1.287 & 4.224 & 0.005 \\
\hline \multicolumn{6}{|c|}{ Deletion in 11q } \\
\hline Yes & vs. no & 1.765 & 1.182 & 2.635 & 0.005 \\
\hline \multicolumn{6}{|c|}{ Leukocyte count (x 10\%) } \\
\hline$\geq 50$ & vs. $<50$ & 3.197 & 2.251 & 4.541 & $<0.001$ \\
\hline \multicolumn{6}{|c|}{ Lymphocyte doubling time } \\
\hline$\leq 12$ months & vs. $>12$ months & 2.178 & 1.632 & 2.907 & $<0.001$ \\
\hline
\end{tabular}

TTFT: time to first treatment; U: unmutated; M: mutated; IGHV: immunoglobulin heavy variable; HR: hazard ratio. 
belonging to subset \#2 should have closer clinical followup, and, in particular, that patients of $\mathrm{m}$-subset \#2 should be considered as high risk despite their formal assignment to the M-CLL group. Correspondingly, this indicates that the BcR IG structure determined by subset assignment may be more important in determining disease biology

Table 4. Time-to-first-treatment (TTF) Cox regression in early stage chronic lymphocytic leukemia (CLL) subset \#2.

\begin{tabular}{|c|c|c|c|c|c|}
\hline \multirow{2}{*}{$\begin{array}{l}\text { Cox regression } \\
\text { TFT }\end{array}$} & \multirow{2}{*}{$\begin{array}{l}\text { Univariable } \\
\text { comparison }\end{array}$} & \multirow[t]{2}{*}{ Hazard ratio } & \multicolumn{2}{|c|}{ 95\% confidence interval } & \multirow[t]{2}{*}{$P$} \\
\hline & & & Lower & Upper & \\
\hline \multicolumn{6}{|c|}{ IGHV analysis group } \\
\hline M-IGHV3-21 & vs. m-IGHV & 1.105 & 0.271 & 4.498 & 0.889 \\
\hline M-subset \#2 & vs.m-IGHV & 4.172 & 2.017 & 8.628 & $<0.001$ \\
\hline U-IGHV & vs. m-IGHV & 4.308 & 3.253 & 5.705 & $<0.001$ \\
\hline \multicolumn{6}{|c|}{ Deletion in $17 p$} \\
\hline Yes & vs. no & 2.305 & 1.273 & 4.172 & 0.006 \\
\hline \multicolumn{6}{|c|}{ Deletion in 11q } \\
\hline Yes & vs.no & 1.749 & 1.169 & 2.615 & 0.006 \\
\hline \multicolumn{6}{|c|}{ Leukocyte count $\left(\mathrm{x} 10^{9} / \mathrm{L}\right)$} \\
\hline$\geq 50$ & vs. $<50$ & 3.209 & 2.260 & 4.556 & $<0.001$ \\
\hline \multicolumn{6}{|c|}{ Lymphocyte doubling time } \\
\hline$\leq 12$ months & s. $>12$ months & 2.181 & 1.633 & 2.912 & $<0.001$ \\
\hline
\end{tabular}

TTFT: time to first treatment; U: unmutated; M: mutated; IGHV: immunoglobulin heavy variable; HR: hazard ratio.

A

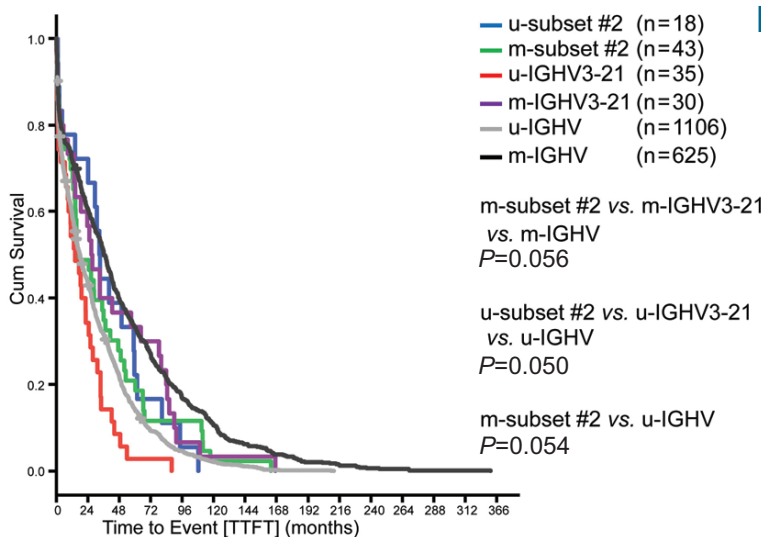

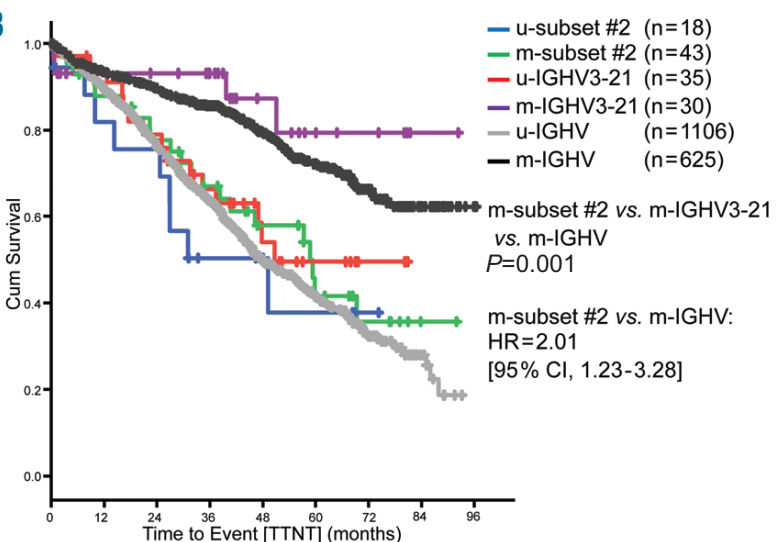

- u-subset \#2 $(n=18)$ - m-subset \#2 $(n=43)$ - u-IGHV3-21 $(n=35)$ $-m-I G H V 3-21(n=30)$ $-\mathrm{U}-\mathrm{IGHV} \quad(n=1106)$ $-\mathrm{m}-\mathrm{IGHV} \quad(\mathrm{n}=625)$ m-subset \#2 vs. m-IGHV3-21 vs. m-IGHV $P=0.020$

m-subset \#2 vs. m-IGHV: . $\mathrm{HR}=1.38$ $[95 \% \mathrm{Cl}, 0.92-2.07]$

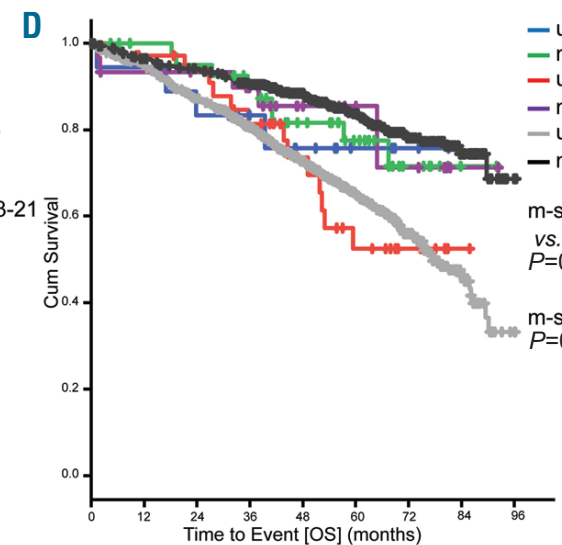

Figure 4. Outcomes of advanced stage chronic lymphocytic leukemia (CLL) cases according to subset \#2 classification and IGHV mutation. (A) Median time-to-firsttreatment (TTFT) u-subset \#2: 32.9 months, m-subset \#2: 16.5 months, u-HV3-21: 14.2 months, m-HV3-21: 26.8 months, u-IGHV: 18.1 months, m-IGHV: 36.3 months. (B) Median time-to-next-treatment (TTNT) u-subset \#2: 49.2 months, m-subset \# 2: 58.7 months, u-HV3-21: 50.6 months, m-HV3-21: not reached, u-IGHV: 47.8 months, m-IGHV: not reached. (C) Median progression-free (PFS) survival u-subset \#2: 21.4 months, m-subset \#2: 35.5 months, u-HV3-21: 31.4 months, mHV3-21: 57.6 months, u-IGHV: 26.7 months, m-IGHV: 54.7 months. (D) Median overall survival (OS) from diagnosis u-subset \#2: not reached, m-subset \#2: not reached, u-HV3-21: not reached, m-HV3-21: not reached, u-IGHV: 77.4 months, m-IGHV: not reached; Cum: cumulative. 
and clinical behavior than IGHV mutational status alone, at least in certain cases.

Subset \#4 is known to have an indolent clinical course and is most frequent among younger patients. ${ }^{15,19}$ According to our findings, subset \#4 had the most favorable prognosis regarding PFS and TTFT compared to the remaining subsets in the early stage CLL population. Furthermore, in the advanced stage CLL analysis, subset \#4 showed a longer TTNT compared to mutated CLL and a longer PFS compared to all other subsets. It is striking that for all time-dependent endpoints in the early stage as well as the advanced stage cohort, the outcome of subset \#4 patients appeared to be even better than M-CLL. This again points to the hypothesis that subset and therefore BcR IG structure may be more relevant in pathogenesis and prognosis than IGHV mutational status.

Subset \#8 has been associated with increased risk of Richter transformation and trisomy $12 .{ }^{35,36}$ In the advanced stage CLL, we observed a higher rate of trisomy 12 and a higher incidence of Richter transformation compared to the other subsets. Subset \#8 showed a shorter PFS compared to subsets \#1 and 2 in advanced stage CLL cases. However, due to the small size of this subgroup population, this could not be tested in a multivariable analysis.

The strength of the current study is also one of its weaknesses, as due to the long follow-up time there was no inclusion of novel agents such as ibrutinib, idelalisib and venetoclax in the treatment schedules. Data of patients treated within clinical studies of novel agents including long follow-up with relevant event numbers and categorized according to the subset classification are not yet available. First analyses indicate that the prognostic power of IGHV mutational status is lost with these agents, ${ }^{37}$ and it would be interesting to see if the subset assignment remains a prognostic factor with these treatments. Furthermore, chemo-immunotherapy remains a possible standard of care for patients without high-risk markers and mutated IGHV. ${ }^{38}$ According to our data, subset assignment by BCR stereotype is relevant for therapy assessment. Therefore, the patients mentioned above should be treated taking into consideration the subset assignment. Future studies will evaluate the relevance of the subset classification regarding the response to novel therapies.

Taken together, we confirmed for the first time in prospective clinical trials that subset \#2 is an independent prognostic marker for shorter TTFT, TTNT and PFS in CLL, regardless of the IGHV mutational status. Therefore, patients with CLL subset \#2 should be closely monitored. Given this, CLL clinical guidelines should include subset \#2 also when mutated as an independent marker for high risk in patients receiving chemo-immunotherapy. Furthermore, our study showed that assignment to stereotyped subsets may have a different impact depending on clinical stage in chemo-immunotherapy settings. Subsets \#1, 2 and 8 had similar prognosis in most clinical outcomes to U-CLL. However, advanced stage CLL subsets $\# 1,2$ and 8 had a longer PFS than U-CLL. Subset \#4 was found to have a better clinical course compared to other subsets in both early and advanced stage CLL and was similar to M-CLL.

For the first time in prospective multicenter clinical trials, subset \# 2 appeared as an independent prognostic factor and subsets \#1, 8 and 4 were found to have a distinctive clinical course and should be proposed for risk stratification of patients.

\section{Funding}

$S J, S S, C S, E T, J B$ received research support by $D F G$ SFB1074 subproject B1 and B2, BMBF PRECISE and EU grant Fire CLL. AA, KS: were supported in part by the framework of action "Postdoctoral Researchers Support" of the operational programme "Research Projects for Postdoctoral Researchers" implemented by the Hellenic Foundation for Research \& Innovation; the project "CRIPIS II ODYSSEUS" funded by the Operational Programme "Competitiveness, Entrepreneurship and Innovation" (NSRF 2014-2020) and cofinanced by Greece and the European Union (European Regional Development Fund)". PG received research support by ERANET TRANSCAN-2 JTC 2016 \#179 NOVEL.

\section{References}

1. Hallek M, Cheson BD, Catovsky D, et al. iwCLL guidelines for diagnosis, indications for treatment, response assessment, and supportive management of CLL. Blood.2018;131(25):2745-2760.

2. Puiggros A, Blanco G, Espinet B. Genetic abnormalities in chronic lymphocytic leukemia: where we are and where we go. Biomed Res Int. 2014;2014:435983.

3. Stilgenbauer S, Schnaiter A, Paschka P, et al. Gene mutations and treatment outcome in chronic lymphocytic leukemia: results from the CLL8 trial. Blood. 2014;123(21):32473254 .

4. Landau DA, Tausch E, Taylor-Weiner AN, et al. Mutations driving CLL and their evolution in progression and relapse. Nature. 2015;526(7574):525-530

5. Damle RN, Wasil T, Fais F, et al. Ig V gene mutation status and CD38 expression as novel prognostic indicators in chronic lymphocytic leukemia. Blood. 1999;94(6):18401847.

6. Hamblin TJ, Davis Z, Gardiner A, et al.
Unmutated Ig $\mathrm{V}(\mathrm{H})$ genes are associated with a more aggressive form of chronic lymphocytic leukemia. Blood. 1999;94(6):18481854.

7. Messmer BT, Albesiano E, Efremov DG, et al. Multiple distinct sets of stereotyped antigen receptors indicate a role for antigen in promoting chronic lymphocytic leukemia. J Exp Med. 2004;200(4):519-525.

8. Rossi D, Gaidano G. Biological and clinical significance of stereotyped B-cell receptors in chronic lymphocytic leukemia. Haematologica. 2010;95(12):1992-1995.

9. Murray F, Darzentas N, Hadzidimitriou A, et al. Stereotyped patterns of somatic hypermutation in subsets of patients with chronic lymphocytic leukemia: implications for the role of antigen selection in leukemogenesis. Blood. 2008;111(3):1524-1533.

10. Agathangelidis A, Darzentas N, Hadzidimitriou A, et al. Stereotyped B-cell receptors in one-third of chronic lymphocytic leukemia: a molecular classification with implications for targeted therapies. Blood. 2012;11(19):4467-4475

11. Stamatopoulos K, Belessi C, Moreno C, et al. Over $20 \%$ of patients with chronic lym- phocytic leukemia carry stereotyped recep tors: Pathogenetic implications and clinical correlations. Blood. 2007;109(1):259-270.

12. Stamatopoulos K, Agathangelidis A Rosenquist R, et al. Antigen receptor stereotypy in chronic lymphocytic leukemia. Leukemia. 2017;31(2):282-291.

13. Bomben R, Dal Bo M, Capello D, et al. Molecular and clinical features of chronic lymphocytic leukaemia with stereotyped B cell receptors: results from an Italian multicentre study. Br J Haematol. 2009; 144(4):492-506

14. Del Giudice I, Chiaretti S, Santangelo S, et al. Stereotyped subset \#1 chronic lymphocytic leukemia: a direct link between B-cell receptor structure, function, and patients' prognosis. Am J Hematol. 2014;89(1):74-82.

15. Baliakas P, Hadzidimitriou A, Sutton L-A, et al. Clinical effect of stereotyped B-cell receptor immunoglobulins in chronic lymphocytic leukaemia: a retrospective multicentre study. Lancet Haematol. 2014;1(2):e74-84.

16. Baliakas P, Agathangelidis A, Hadzidimitriou A, et al. Not all IGHV3-21 chronic lymphocytic leukemias are equal: prognostic considerations. Blood. 2015;125(5):856-859. 
17. Bomben R, Dal Bo M, Capello D, et al. Comprehensive characterization of IGHV321-expressing B-cell chronic lymphocytic leukemia: an Italian multicenter study. Blood. 2007;109(7):2989-2998

18. Jeromin S, Haferlach C, Dicker F, et al. Differences in prognosis of stereotyped IGHV3-21 chronic lymphocytic leukaemia according to additional molecular and cytogenetic aberrations. Leukemia. 2016; 30(11):2251-2253.

19. Xochelli A, Baliakas P, Kavakiotis I, et al. Chronic Lymphocytic Leukemia with Mutated IGHV4-34 Receptors: Shared and Distinct Immunogenetic Features and Clinical Outcomes. Clin Cancer Res. 2017; 23(17):5292-5301.

20. Gounari M, Ntoufa S, Apollonio B, et al. Excessive antigen reactivity may underlie the clinical aggressiveness of chronic lymphocytic leukemia stereotyped subset \#8. Blood. 2015;125(23):3580-3587

21. Hoechstetter MA, Busch R, Eichhorst B, et al. Early, risk-adapted treatment with fludarabine in Binet stage A chronic lymphocytic leukemia patients: results of the CLL1 trial of the German CLL study group. Leukemia. 2017;31(12):2833-2837.

22. Hallek M, Fischer K, Fingerle-Rowson G, et al. Addition of rituximab to fludarabine and cyclophosphamide in patients with chronic lymphocytic leukaemia: a randomised, open-label, phase 3 trial. Lancet. 2010;376(9747):1164-1174.

23. Fischer K, Bahlo J, Fink AM, et al. Long-term remissions after FCR chemoimmunotherapy in previously untreated patients with CLL: updated results of the CLL8 trial. Blood. 2016;127(2):208-215.

24. Eichhorst B, Fink A-M, Bahlo J, et al. First- line chemoimmunotherapy with bendamustine and rituximab versus fludarabine, cyclophosphamide, and rituximab in patients with advanced chronic lymphocytic leukaemia (CLL10): an international, openlabel, randomised, phase 3 , non-inferiority trial. Lancet Oncol. 2016;17(7):928-942

25. Goede V, Fischer K, Busch R, et al. Obinutuzumab plus chlorambucil in patients with CLL and coexisting conditions. N Engl J Med. 2014;370(12):11011110.

26. Döhner H, Stilgenbauer S, Benner A, et al. Genomic aberrations and survival in chronic lymphocytic leukemia. N Engl J Med. 2000;343(26):1910-1916.

27. Kröber A, Seiler T, Benner A, et al. V(H) mutation status, CD38 expression level, genomic aberrations, and survival in chronic lymphocytic leukemia. Blood. 2002; 100(4):1410-1416.

28. Thunberg U, Johnson A, Roos G, et al $\mathrm{CD} 38$ expression is a poor predictor for $\mathrm{VH}$ gene mutational status and prognosis in chronic lymphocytic leukemia. Blood. 2001 97(6):1892-1894

29. Strefford JC, Sutton L-A, Baliakas P, et al. Distinct patterns of novel gene mutations in poor-prognostic stereotyped subsets of chronic lymphocytic leukemia: the case of SF3B1 and subset \#2. Leukemia. 2013; 27(11):2196-2199.

30. Hallek M, Wanders L, Ostwald $M$, et al. Serum beta(2)-microglobulin and serum thymidine kinase are independent predictors of progression-free survival in chronic lymphocytic leukemia and immunocytoma. Leuk Lymphoma. 1996;22(5-6):439-447.

31. Kaplan EL, Meier P. Nonparametric estimation from incomplete observations. J. Amer.
Statist. Assn. 1958;53(282):457-481.

32. Tobin G, Thunberg U, Johnson A, et al Somatically mutated Ig V(H)3-21 genes characterize a new subset of chronic lymphocytic leukemia. Blood. 2002;99(6):2262-2264.

33. Thorsélius M, Kröber A, Murray F, et al Strikingly homologous immunoglobulin gene rearrangements and poor outcome in VH3-21-using chronic lymphocytic leukemia patients independent of geographic origin and mutational status. Blood. 2006;107(7):2889-2894.

34. Ghia EM, Jain S, Widhopf GF, et al. Use of IGHV3-21 in chronic lymphocytic leukemia is associated with high-risk disease and reflects antigen-driven, post-germinal center leukemogenic selection. Blood. 2008; 111(10):5101-5108.

35. Rossi D, Spina V, Bomben R, et al Association between molecular lesions and specific B-cell receptor subsets in chronic lymphocytic leukemia. Blood. 2013;121(24): 4902-4905.

36. Rossi D, Spina V, Cerri M, et al. Stereotyped $\mathrm{B}$-cell receptor is an independent risk factor of chronic lymphocytic leukemia transformation to Richter syndrome. Clin Cancer Res. 2009;15(13):4415-4422

37. Kipps TJ, Fraser G, Coutre S, et al Unmutated IGHV is not an adverse predictor of outcome to therapy with ibrutinib in patients with chronic lymphocytic leukemia/small lymphocytic lymphoma (CLL/SLL). Cancer Res 2017;77(13 Suppl):abstract CT158.

38. Wierda WG, Byrd JC, Abramson JS, et al NCCN guidelines insights: chronic lymphocytic leukemia/small lymphocytic lymphoma, version 2.2019. J Natl Compr Canc Netw. 2019;17(1):12-20. 